\title{
PRESIÓN ARTERIAL Y OBESIDAD ABDOMINAL EN PERSONAS DE LA UNIVERSIDAD DEL QUINDÍO.
}

\author{
BLOOD PRESSURE AND ABDOMINAL OBESITY IN PEOPLE OF THE \\ QUINDIO'S UNIVERSITY
}

\author{
Olga Alicia Nieto-Cárdenas ${ }^{1 *}$ \\ ${ }^{1}$ Programa de Medicina, Facultad de Ciencias de la Salud, Universidad del Quindío. \\ oanieto@uniquindio.edu.co \\ http://orcid.org/0000-0002-0909-3528 \\ *Correspondencia del Autor: Facultad de ciencias de la Salud, Universidad del Quindío. \\ Carrera 15 calle 12 Norte.Correo electrónico: oanieto@uniquindio.edu.co
}

\section{RESUMEN}

Objetivo. Describir dos indicadores de riesgo cardiovascular (RCV), en una población universitaria durante 4 años.

Material y método: Se llevó a cabo un estudio descriptivo durante 4 años de la presión arterial y el perímetro abdominal en la comunidad universitaria. Se describieron variables en promedio, desviación estándar e intervalos de confianza, se hizo análisis comparativo por sexo; se realizaron análisis de varianza y Chi cuadrado y se consideró un valor de $\mathrm{p}<0,05$ para diferencias estadísticamente significativas.

Resultados: Participaron en promedio 402 personas semestralmente, $66,57 \%$ estudiantes, $17,57 \%$ administrativos, 7,18\% los docentes y otros 7,03\%. Mujeres $46,01 \%$ y hombres $53,99 \%$. El promedio de edad fue de 28,79 años. El perímetro abdominal promedio fue de 82,37 centímetros y la tensión arterial de 113/73 en promedio.

Se encontraron relaciones estadísticamente significativas en la participación de los estudiantes es en su mayoría masculino con $36,91 \%$ y un $29,63 \%$ femenino. Y la de los administrativos es en su mayoría femenina con un $10,26 \%$ y masculino $7,31 \%$.

El perímetro abdominal promedio presenta un aumento progresivo y una relación estadísticamente significativa (valor de $\mathrm{p}<0,05$ ) con edad, sexo y estamento.

Se encontró una relación estadísticamente significativa con el sexo, la edad y la presión arterial sistólica y diastólica (valor de $\mathrm{p}<0,05$ ).

Discusión y conclusión: El perímetro abdominal y la presión arterial se encuentran en promedio en normalidad, sin embargo, el perímetro abdominal aumenta progresivamente y se encontró un 15,9\% de RCV en la comunidad universitaria con estos indicadores.

Palabras clave: Gestión de riesgo; presión arterial; obesidad abdominal.

Cómo citar:

Nieto-Cárdenas, Olga Alicia (2020) PRESIÓN ARTERIAL Y OBESIDAD ABDOMINAL EN PERSONAS DE LA UNIVERSIDAD DEL QUINDÍO. Revista de Investigaciones Universidad del Quindio, vol. 32(1), pp.7-15. https://doi.org/10.33975/riuq.vol32n1.368 


\begin{abstract}
Objective. Describe two cardiovascular risk indicators (CVR), in a university population for 4 years.

Material and method. A retrospective descriptive study is carried out during 4 years of blood pressure and abdominal perimeter in the university community. Variables in average, standard deviation and confidence intervals were described, a comparative analysis was made by sex; analysis of variance and Chi square were performed and a value of $\mathrm{p}<0.05$ was considered for statistically significant differences.

Results. On average 402 people participated semiannually, $66.57 \%$ students, $17.57 \%$ administrative, $7.18 \%$ teachers and other $7.03 \%$. Women $46.01 \%$ and men $53.99 \%$. The average age was 28.79 years. The average abdominal perimeter was 82.37 centimeters and the blood pressure 113/73 on average.

Statistically significant relationships were found in student participation is mostly male with $36.91 \%$ and $29.63 \%$ female. And the administrative staff is mostly female with $10.26 \%$ and male $7.31 \%$.

The average abdominal perimeter presents a progressive increase and a statistically significant relationship (value of $\mathrm{p}<0,05$ ) with age, sex and status.

A statistically significant relationship was found with sex, age and systolic and diastolic blood pressure ( $\mathrm{p}$ value $<0,05)$.
\end{abstract}

Discussion and conclusion.

The abdominal perimeter and blood pressure are on average in normal limits, however, the abdominal perimeter increases progressively and $15.9 \%$ CVR was found in the university community with these indicators.

Keywords: Risk management; arterial pressure; obesity Abdominal.

\section{INTRODUCCIÓN}

El concepto de riesgo como la probabilidad que ocurra un efecto adverso que afecte nuestra salud y vida ha orientado la posibilidad de prevenir la enfermedad. Y la idea de trabajar en la detección del riesgo como una opción de prevención, es una constante en la historia de la salud pública.

En el desarrollo del sistema general de seguridad social en salud (SGSSS) en Colombia, precisa la importancia de los conceptos de detección temprana del riesgo e inducción a la demanda (1) y posteriormente se llega al concepto de gestión del riesgo.

El Plan Decenal de Salud Pública, PDSP, 20122021 (2), concibe el riesgo en salud como "la probabilidad de ocurrencia de un evento no deseado, evitable y negativo para la salud del individuo, que puede ser también el empeoramiento de una condición previa o la necesidad de requerir más consumo de bienes y servicios que hubiera podido evitarse".

El evento es la ocurrencia de la enfermedad o su evolución desfavorable y sus causas son los diferentes factores asociados. El riesgo en salud a su vez puede clasificarse como primario si se refiere a la probabilidad de aparición de nueva morbilidad (incidencia), o como técnico si alude a la probabilidad de "ocurrencia de eventos derivados de fallas de atención en los servicios de salud y de la mayor carga de enfermedad por mortalidad evitable y discapacidad" (2).

"La comprensión del concepto de riesgo en salud, el conocimiento de sus principales determinantes y de los mecanismos a través de los cuales se genera, es fundamental para su identificación y el desarrollo de estrategias para su prevención, mitigación y superación (3)."

En el aseguramiento en salud, se define el sistema de administración de riesgos y el riesgo desde la perspectiva epidemiológica se relaciona con el riesgo desde la perspectiva empresarial, entendido este último como la probabilidad de generación de pérdidas económicas por la ocurrencia de un evento adverso derivado de las actividades propias del negocio (4).

Más recientemente el SGSSS llega al concepto de rutas integrales de atención en salud (RIAS), con el objetivo de integrar a todos los actores del sistema para una adecuada gestión del riesgo en 
salud (5).

\section{El concepto de riesgo cardiovascular}

La probabilidad de enfermar y morir por un evento cardiovascular se reconoce como riesgo cardiovascular (RCV) y se ha medido tradicionalmente con las tablas de Framingham, que utilizan un método de puntuación con las siguientes variables: edad (35-74 años), sexo, HDL colesterol, colesterol total, presión arterial sistólica, tabaquismo (sí/no), diabetes (sí/no), e hipertrofia ventricular izquierda (HVI) (sí/no), con lo cual es posible calcular el riesgo coronario a los 10 años que incluye: angina estable, infarto de miocardio (IAM) y muerte coronaria (6).

Posteriormente aparece el concepto de síndrome metabólico (SM), que implica al menos un aumento del doble del riesgo de eventos cardiovasculares y el pronóstico es peor después de cualquier evento coronario agudo; asimismo, la presencia de SM predice el desarrollo de insuficiencia cardiaca crónica y un incremento en la mortalidad por la misma (7). Según el concepto de la Federación internacional de Diabetes, el SM constituye una entidad de origen multifactorial, que aumenta el riesgo de desarrollar diabetes, el cual se caracteriza por la presencia de alteraciones como resistencia a la insulina, hiperinsulinismo, obesidad, alteraciones del metabolismo de la glucosa, hipertensión arterial (HTA) y dislipidemia 8).

Para la población latinoamericana, se utilizan los criterios establecidos por la Asociación Latinoamericana de Diabetes (ALAD) (9), que son: Obesidad abdominal (perímetro abdominal $>94$ $\mathrm{cm}$ en hombres y $>88 \mathrm{~cm}$ en mujeres); triglicéridos elevados: $\geq 150 \mathrm{mg} / \mathrm{dL}$ o en tratamiento hipolipemiante especifico; Colesterol HDL (HDLc) bajo: $<40 \mathrm{mg} / \mathrm{dL}$ en hombres y $<50 \mathrm{mg} / \mathrm{dL}$ en mujeres; presión Arterial alta: $\geq 130 / 85 \mathrm{mmHg}$ o en tratamiento antihipertensivo; alteración en la glicemia: glicemia en ayunas $>100 \mathrm{mg} / \mathrm{dL}$ o Intolerancia Oral a los Carbohidratos (IOC) o Diabetes Mellitus (DM) diagnosticada.

\section{Los indicadores de RCV}

El instructivo para calcular el riesgo cardiovascular del Ministerio de Salud y Protección Social (MSPS) (10), incluye como indicadores la obesidad abdominal, el sobrepeso y obesidad, el riesgo cardiovascular (medido a través de las tablas de Framingham) y el riesgo de diabetes (medido a través del findrisk).

Según la Guía de la práctica clínica (GPC) para manejo de la hipertensión arterial se reconocen como cifras normales 120/80 mmHg de presión arterial; de "pre-hipertensión" o "presión normal-alta", valores de presión arterial sistólica entre 120-139 mmHg y de diastólica entre 80-89 mmHg; "sospecha de hipertensión arterial", cuya medición de consultorio indique presión arterial sistólica (PAS) 135-150 mmHg o presión arterial diastólica (PAD) 85-95 mmHg (11). Y de acuerdo con los indicadores antropométricos, en Colombia se identifican como de riesgo cifras de perímetro abdominal $>90 \mathrm{~cm}$ en hombres y $>80 \mathrm{~cm}$ en mujeres (12).

\section{EI trabajo en jornadas de salud pública}

Es el trabajo en salud pública durante un día o jornada específica, en el cual los agentes de salud se dedican a atender a las personas de acuerdo a un riesgo y no por demanda espontánea como ocurre en una consulta.

Posteriormente, en SGSSS, el trabajo en jornadas está descrito en el contexto del Plan de Intervenciones colectivas (PIC), se definen de la siguiente manera (13): "Las jornadas de salud son una modalidad de prestación de servicio definida en la Res. 2003 de 2014 como "Conjunto de actividades en salud que se desarrollan de manera puntual y esporádicamente en infraestructuras físicas no destinadas a la atención en salud, o infraestructuras físicas de salud en áreas de difícil acceso, que no cuentan con servicios quirúrgicos habilitados, o actividades en salud que se prestan en unidades móviles aéreas, fluviales, marítimas o terrestres que requieren para su funcionamiento 
recurso humano, equipos, materiales y medicamentos necesarios, para beneficio de la población del área de influencia."

\section{METODOLOGÍA}

\section{Tipo de estudio}

Se llevó a cabo un estudio descriptivo, durante los años 2015-2018 de la presión arterial y el perímetro abdominal de la comunidad universitaria.

\section{Población y sujetos de estudio}

La población sujeto fue la comunidad de la Universidad del Quindío, que ha participado voluntariamente durante estos años en la jornada de detección de riesgo cardiovascular: estudiantes, docentes, administrativos y otros, que circulan por el campus de Armenia. Según informe de la oficina de planeación, la población estudiantil de la modalidad presencial en Armenia ha venido en aumento y se registra en este periodo, 7.996 estudiantes en el año 2015 y 9.545 estudiantes en el año 2018.

La Jornada de detección de RCV se llevó a cabo semestralmente, con estudiantes de medicina interna, quienes se ubicaron simultáneamente en 10 sitios de mayor tránsito en la Universidad con carteles que motivaran a las personas para hacerse dos mediciones: toma de presión arterial y perímetro abdominal.

Las personas que presentaron cifras de hipertensión arterial o pre-hipertensión", y quienes presentaron cifras de obesidad abdominal, es decir, los indicadores por encima de los límites, se remitieron al Centro de Salud para continuar con la valoración y de ser necesario, para consulta de RCV o consulta nutricional y fueron motivados para mejorar su actividad física.

Se garantizó la confidencialidad en el manejo de la información para el estudio. Sin embargo, las variables de identificación fueron muy importantes para la detección y remisión de los participantes para la consulta de RCV.
Las variables incluidas en el estudio fueron: nombre, número de identificación, teléfono, afiliación a EPS, estamento (estudiante, docente, administrativo, visitante, otro), facultad, dependencia o programa, edad (años), sexo (f o m), tensión Arterial (sistólica y diastólica en $\mathrm{mmHg}$ ), perímetro abdominal $(\mathrm{cm})$ y remitido (Centro de salud o a su EPS).

\section{Análisis y procesamiento de la información}

Para efectos del presente trabajo, se hizo acopio de la información generada durante las jornadas desde el año 2015 al 2018, en Excel ${ }^{\circledR}$, se depuraron las tablas y se procesó la información en

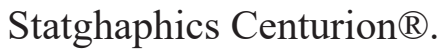

Se describieron las variables en promedio \pm desviación estándar e intervalos de confianza las numéricas y en frecuencias las categóricas.

Se compararon las variables entre los diferentes grupos de la población universitaria: estudiantes, docentes, administrativos y otros; y se establecieron diferencias significativas cuando el valor de $\mathrm{p} \leq 0,05$, con una prueba de Chi cuadrado cuando las variables fueron categóricas, o con una prueba de ANOVA (análisis de varianza) cuando las variables eran numéricas.

\section{RESULTADOS}

Se llevaron a cabo 8 jornadas de detección de RCV entre los años 2015 a 2018, con la participación por estamento: administrativos, docentes, estudiantes y otro.

Participaron 3.218 personas, un promedio de 402 personas por semestre. Los estudiantes, tuvieron participación mayoritaria con el 66,55\%; 17,56\% administrativos, 7,18\% docentes, 7,06\% otro (visitantes, particulares, egresados, contratistas) y $1,65 \%$ no registrado.

El promedio de edad fue de 28,79 años con un IC95\% de 28,32 a 29,25. Allí están incluidos todos los estamentos universitarios. 
El perímetro abdominal promedio fue de 82,37 centímetros con un IC95\% de 81,97 82,77 (rango $57-131 \mathrm{~cm})$.

La presión arterial estuvo en promedio en 113/73. La presión sistólica tuvo un IC95\% de 133,33 a 114,16 y la diastólica un IC95\% de 73,20 a 73,85.
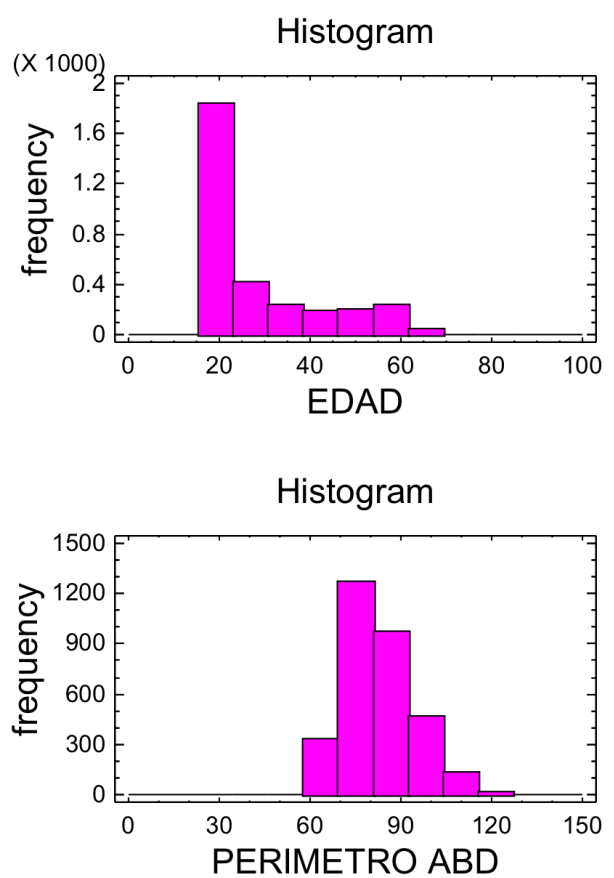

Figura 1 y 2. Distribución de las variables edad y perímetro abdominal en toda la población
Las jornadas semestrales se describen la primera del año con A y la segunda con B. En el primer semestre del 2015 participaron 452 personas y en el segundo participaron 460 personas; en el 2016A participaron 399 y 2016B acudieron 397 personas; 2017A participaron 397 y 2017B participaron 327 personas; 2018A acudieron 359 y en 2018B participaron 427 personas.

La distribución por sexo de la población participante fue $46,01 \%$ mujeres y $53,99 \%$ hombres.

La identificación del RCV, por los niveles de la tensión arterial o el perímetro abdominal, hizo que se remitieran 498 personas $(15,93 \%)$ para consulta de riesgo cardiovascular al Centro de Salud.

La afiliación a la seguridad social, permitió identificar 58 diferentes EPS, entre las cuales las 10 primeras son: Cafesalud, Nueva EPS, Coomeva, Medimás, Sura, Sanitas, S.O.S, Cosmitet, Saludcoop, Asmet Salud, para un 77,34\%. Si se incluyen Uniquindío y ninguna, el porcentaje alcanza un $80,07 \%$, el porcentaje restante se divide entre otras EPS.

Al comparar por sexo, la participación en las jornadas no tuvo diferencias significativas.

Tabla 1. Comparación por estamento y por sexo.

\begin{tabular}{|c|c|c|c|c|}
\hline VARIABLE & CATEGORÍAS & FRECUENCIA & $\begin{array}{c}\text { PORCENTAJE } \\
(\%)\end{array}$ & $\begin{array}{l}\text { VALOR DE } p \\
\text { (Chi cuadrado) }\end{array}$ \\
\hline $\begin{array}{l}\text { Participación en las jor- } \\
\text { nadas por estamento }\end{array}$ & $\begin{array}{l}\text { Administrativo } \\
\text { Docente } \\
\text { Estudiante } \\
\text { Otro } \\
\text { No registrado }\end{array}$ & $\begin{array}{c}565 \\
231 \\
2141 \\
54 \\
227\end{array}$ & $\begin{array}{c}17,57 \\
7,18 \\
66,57 \\
1,65 \\
7,03\end{array}$ & $<0,001$ \\
\hline $\begin{array}{c}\text { Participación en jornadas } \\
\text { por Sexo } \\
\text { Femenino } \\
46,99 \%\end{array}$ & $\begin{array}{l}2015 \mathrm{~A} \\
2015 \mathrm{~B} \\
2016 \mathrm{~A} \\
2016 \mathrm{~B} \\
2017 \mathrm{~A} \\
2017 \mathrm{~B} \\
2018 \mathrm{~A} \\
2018 \mathrm{~B}\end{array}$ & $\begin{array}{l}189 \\
212 \\
176 \\
169 \\
187 \\
158 \\
171 \\
218\end{array}$ & $\begin{array}{l}5,87 \\
6,59 \\
5,47 \\
5,25 \\
5,81 \\
4,91 \\
5,31 \\
6,77\end{array}$ & 0,1265 \\
\hline
\end{tabular}




\begin{tabular}{|c|c|c|c|c|}
\hline Masculino & $2015 \mathrm{~A}$ & 262 & 8,14 & \\
\hline \multirow[t]{7}{*}{$54,01 \%$} & 2015 B & 249 & 7,74 & \\
\hline & $2016 \mathrm{~A}$ & 223 & 6,93 & \\
\hline & 2016 B & 228 & 7,09 & \\
\hline & $2017 \mathrm{~A}$ & 212 & 6,59 & \\
\hline & 2017 B & 167 & 5,19 & \\
\hline & $2018 \mathrm{~A}$ & 188 & 5,84 & \\
\hline & $2018 \mathrm{~B}$ & 209 & 6,49 & \\
\hline \multirow{8}{*}{ Remitido por jornadas } & $2015 \mathrm{~A}$ & NR & NR & $<0,001$ \\
\hline & 2015 B & 48 & 1,60 & \\
\hline & $2016 \mathrm{~A}$ & 95 & 3,17 & \\
\hline & 2016 B & 132 & 4,40 & \\
\hline & $2017 \mathrm{~A}$ & 48 & 1,60 & \\
\hline & 2017 B & 69 & 2,30 & \\
\hline & $2018 \mathrm{~A}$ & 58 & 1,93 & \\
\hline & 2018 B & 32 & 1,07 & \\
\hline \multirow[t]{4}{*}{ Remitido por estamento } & Administrativo & 158 & 5,27 & $<0,001$ \\
\hline & Docente & 73 & 2,39 & \\
\hline & Estudiante & 175 & 5,87 & \\
\hline & Otro & 75 & 2,49 & \\
\hline \multirow[t]{3}{*}{ Remitido por sexo } & Remitido CS & 183 & 6,1 & $<0,001$ \\
\hline & Remitido EPS & 1 & 0,03 & \\
\hline & No remitido & 1013 & 33,76 & \\
\hline Femenino & No registrado & 189 & 6,30 & \\
\hline \multirow{4}{*}{ Masculino } & Remitido CS & 295 & 9,84 & \\
\hline & Remitido EPS & 3 & 0,10 & \\
\hline & No remitido & 1054 & 35,13 & \\
\hline & No registrado & 262 & 8,73 & \\
\hline
\end{tabular}

Si se encuentran diferencias significativas por sexo entre los estamentos participantes (valor de $\mathrm{p}<0,05$ ), la participación de los estudiantes es en su mayoría masculino con $36,91 \%$ y un $29,63 \%$ femenino. Y la de los administrativos (17,57\%), es en su mayoría femenina con un $10,26 \%$ y masculino $7,31 \%$.

Se identificó RCV o metabólico en 478 personas $(15,94 \%)$, es decir que los indicadores registrados estaban por encima de los límites normales; las cuales fueron remitidas a consulta de riesgo cardiovascular al Centro de Salud de la Universidad del Quindío y 4 personas $(0,13 \%)$ a su propia EPS.

La remisión por sexo si tuvo diferencias significativas (valor de $\mathrm{p}<0,05$ ), siendo mayor la remisión de hombres $(9,94 \%)$ que de mujeres $(6,13 \%)$, es decir se identificó mayor porcentaje de hombres con RCV y metabólico que de mujeres.

El perímetro abdominal presenta una relación estadísticamente significativa (valor de $\mathrm{p}<0,05$ ) con edad, sexo masculino y estamento; mayor edad en administrativos y mayor perímetro abdominal en los docentes. También se observa un aumento progresivo del perímetro abdominal durante las diferentes jornadas.

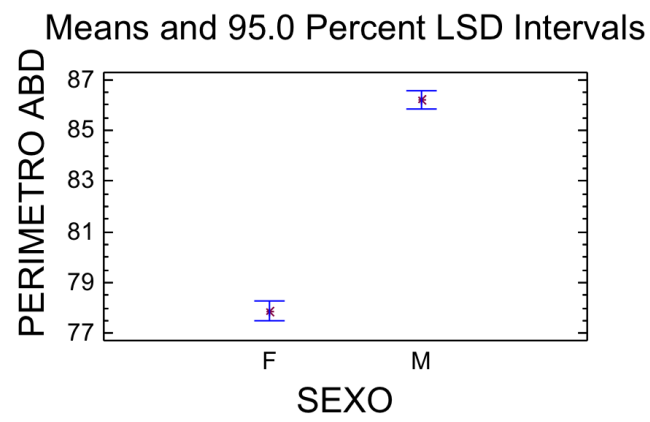

Figura 3. Diferencias de perímetro abdominal por sexo. 
Tabla 2. Análisis de varianza por estamento, sexo y remisión

\begin{tabular}{|c|c|c|c|}
\hline VARIABLE & CATEGORÍAS & PROMEDIO \pm DE & $\begin{array}{l}\text { VALOR DE } p \\
\text { (ANOVA) }\end{array}$ \\
\hline $\begin{array}{c}\text { EDAD por estamento } \\
\text { En años }\end{array}$ & $\begin{array}{c}\text { Administrativo } \\
\text { Docente } \\
\text { Estudiante } \\
\text { Otro } \\
\text { No registrado }\end{array}$ & $\begin{array}{c}4,73 \pm 11,65 \\
44,46 \pm 12,00 \\
21,28 \pm 4,56 \\
43,68 \pm 13,42 \\
29,49 \pm 14,85\end{array}$ & $<0,001$ \\
\hline $\begin{array}{l}\text { Perímetro abdominal } \\
\text { por estamento } \\
\text { En centímetros }\end{array}$ & $\begin{array}{c}\text { Administrativo } \\
\text { Docente } \\
\text { Estudiante } \\
\text { Otro } \\
\text { No registrado }\end{array}$ & $\begin{array}{l}88,44 \pm 12,00 \\
\mathbf{9 1 , 0 0} \pm \mathbf{1 0 , 6 3} \\
79,10 \pm 10,09 \\
89,22 \pm 11,46 \\
82,98 \pm 11,44\end{array}$ & $<0,001$ \\
\hline Perímetro abdominal por sexo & $\begin{array}{l}\text { Femenino } \\
\text { Masculino }\end{array}$ & $\begin{array}{l}77,87 \pm 10,08 \\
86,20 \pm 11,45\end{array}$ & $<0,001$ \\
\hline $\begin{array}{c}\text { Tensión arterial Diastólica } \\
\text { Por estamento } \\
\text { (TAD) En mmHg }\end{array}$ & $\begin{array}{l}\text { Administrativo } \\
\text { Docente } \\
\text { Estudiante } \\
\text { Otro } \\
\text { No registrado }\end{array}$ & $\begin{array}{c}75,43 \pm 10,15 \\
76,44 \pm 9,99 \\
72,20 \pm 8,88 \\
76,93 \pm 10,39 \\
78,39 \pm 9,39\end{array}$ & $<0,001$ \\
\hline $\begin{array}{c}\text { Tensión arterial Sistólica por esta- } \\
\text { mento } \\
\text { (TAS) En mmHg }\end{array}$ & $\begin{array}{l}\text { Administrativo } \\
\text { Docente } \\
\text { Estudiante } \\
\text { Otro } \\
\text { No registrado }\end{array}$ & $\begin{array}{l}116,62 \pm 13,26 \\
118,27 \pm 12,60 \\
111,82 \pm 10,75 \\
119,39 \pm 14,12 \\
117,35 \pm 13,17\end{array}$ & $<0,001$ \\
\hline $\begin{array}{l}\text { Remitido por edad } \\
\text { Edad en años }\end{array}$ & $\begin{array}{c}\text { Remitido CS (478) } \\
\text { Remitido EPS (4) } \\
\text { No remitido (2067) } \\
\text { No registrado (451) }\end{array}$ & $\begin{array}{l}38,63 \pm 15,46 \\
38,00 \pm 17,94 \\
27,18 \pm 12,28 \\
26,92 \pm 12,22\end{array}$ & $<0,001$ \\
\hline
\end{tabular}

Se llevó a cabo una regresión múltiple del perímetro abdominal y se encontró relación estadísticamente significativa con el sexo, la edad y la presión arterial sistólica (PAS) y diastólica (PAD) (valor de $\mathrm{p}<0,05$ ).

En la regresión múltiple para la tensión arterial diastólica, se encontró relación estadísticamente significativa con PAS, sexo, estamento y edad.

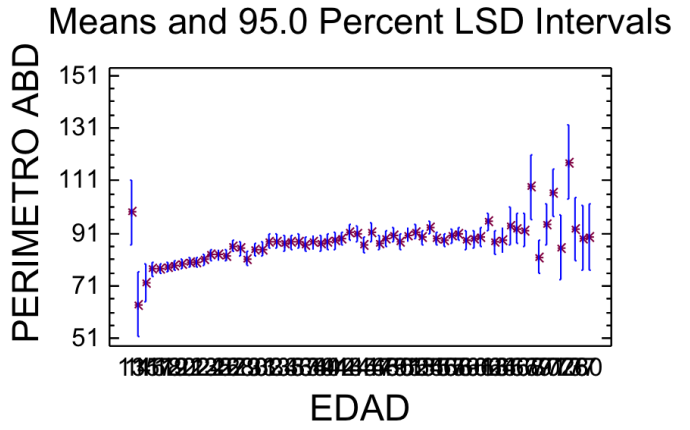

Figura 4. Relación perímetro abdominal con edad. 


\section{DISCUSIÓN}

Los indicadores de riesgo cardiovascular y metabólico tales como el perímetro abdominal y la presión arterial que se describen en el presente estudio usualmente se tienen en cuenta en poblaciones de adultos mayores, por ejemplo, la Escala de Framingham identifica el riesgo en mayores de 30 años, sin embargo, cada vez más se identifica la necesidad de su monitoreo como un continuo desde tempranas edades.

En esta comunidad universitaria, con mayor participación de estudiantes, las cifras promedio de la presión arterial promedio y el perímetro abdominal, se encuentran en promedio en normalidad, sin embargo, el perímetro abdominal muestra una tendencia creciente durante los 4 años del estudio. Este aumento del perímetro abdominal, es un indicador importante de resistencia la insulina (14), de resistencia a la leptina (15) y de síndrome metabólico.

Según la guía de la práctica clínica de la ALAD 2019 (16), "El Síndrome Metabólico (SM) es un grupo de factores biológicos caracterizados por obesidad, resistencia a la insulina, hipertensión y dislipidemia que juntos culminan en un incremento del riesgo de desarrollar diabetes tipo 2 y enfermedad cardiovascular; siendo ambas principales causas de muerte a nivel mundial. La presencia de SM incrementa hasta en cinco veces el riesgo de diabetes mellitus tipo 2 (DM2) y tres veces el riesgo de enfermedad cardiovascular (ECV).

Se encontró un 15,9\% de RCV y metabólico en la comunidad universitaria con estos indicadores y las personas fueron remitidas al Centro de Salud de la Universidad. Según la ALAD “el diagnóstico temprano y oportuno del SM en la población aparentemente sana es una de las herramientas para establecer medidas de prevención ante el riesgo de desarrollo de otras comorbilidades, la progresión hacia complicaciones crónicas y por consecuente evitar una mala calidad de vida del paciente (16)."
En esta recomendación de hacer una detección cada vez más temprana del SM y del RCV, reside la importancia de estos resultados como una contribución a la construcción de una Universidad Saludable.

En el año 2015 se llevó a cabo el proyecto de investigación, denominado: "Prevalencia de factores de Riesgo Cardiovascular en esta misma comunidad universitaria (17), en el cual se encontró que el riesgo de presentar un episodio cardiovascular en los próximos 10 años en una población universitaria con un promedio de edad de 33,64 años, fue de 2,54\% según la escala de Framingham. El 2,3\% de los participantes presentaron riesgo medio y alto, que corresponden al grupo de administrativos. Y se encontró que el factor de RCV que más tempranamente se altera es la relación cintura cadera (un indicador antropométrico nutricional), seguido del perímetro abdominal (un indicador metabólico de RCV) y por último se evidencia el indicador de RCV medido con Framingham (17), lo cual coincide con el presente estudio.

En un contexto latinoamericano, el estudio de Gnatiuc (18) y colaboradores en un estudio de más de 150.000 personas, encontró que la obesidad abdominal está fuertemente asociada con la mortalidad en la población mexicana.

Algunas recomendaciones pueden retomarse en una política sobre Universidad Saludable en la Universidad del Quindío: La promoción de la actividad y el ejercicio físico, la promoción de una alimentación saludable, en la cual sería importante incluir la prohibición y control del expendio de bebidas azucaradas y comestibles en paquetes y el estímulo del expendio de frutas, por ejemplo. La promoción de ambientes libres de humo y la prohibición de consumo de cigarrillo en el entorno universitario. Y el fortalecimiento del programa de prevención del riesgo cardiovascular para la población universitaria en la consulta de la IPS de la Universidad. 


\section{CONCLUSIONES}

Se identificó RCV o metabólico en 15,94\% de los participantes, al encontrarse fuera de los niveles de la tensión arterial o el perímetro abdominal. El indicador de perímetro abdominal subió progresivamente durante los 4 años del estudio en esta comunidad universitaria.

\section{AGRADECIMIENTOS}

A la Universidad del Quindío, a todas y cada una de las personas que han participado para identificar su propio RCV, al Centro de Salud de la Universidad y a su coordinadora, la Dra. María Victoria Salazar. Especial reconocimiento a los estudiantes de Salud del Adulto, quienes en estos años, en el espacio de Salud Pública han llevado a cabo esta jornada como parte de su proceso de formación en atención primaria en salud, promoción de la salud y prevención del riesgo.

\section{REFERENCIAS}

1. MSPS. Resolución 412 de 2000.

2. MSPS. Plan Decenal de Salud 2012-2021. MSPS. Resolución 1841 de 2013, p. 147.

3. Gestión integral del riesgo en salud. Comité Ampliado MSPS. 2015.

4. MSPS. Sistema de administración de riesgos. Resolución 1740 de 2008.

5. MSPS. Ruta integral de atención para la promoción y mantenimiento de la salud. Resolución 3280 de 2018

6. Alvarez Cosmea. Las tablas de riesgo cardiovascular. Medifam. 2001; 11: 122-139.

7. Kassi E, Pervanidou P, Kaltsas G, Chrousos G. Metabolic syndrome: definitions and controversies. BMC Medicine. 2011; 9:48-60. https://doi.org/10.1186/1741-7015-9-48

8. Federación Internacional de Diabetes. El I Congreso Internacional sobre Prediabetes y el Síndrome Metabólico. Diabetes Voice. 2005; 50:1-3.

9. Asociación Latinoamericana de Diabetes. Epidemiología, Diagnóstico, Control, Prevención y Tratamiento del Síndrome Metabólico en Adultos. Revista ALAD. 2010; 18:25-44.

10. MSPS. Instructivo conoce tu riesgo. Finnish-escore Framingham. Tablas estratificación OMS. 2019. $22 \mathrm{pp}$.

11. Guía de la práctica clínica para el manejo de la hipertensión arterial primaria. SGSSS. Guía No. 18. Segunda edición. Instituto de Evaluación Tecnológica en Salud, IETS. MSPS. 2017.

12. MSPS. Indicadores antropométricos. Resolución 2465 de 2016.

13. MSPS. Orientaciones a las jornadas de salud. Dirección de Promoción y Prevención. 2015.

14. Pollak C. F. Resistencia a la insulina: verdades y controversias. Rev. Méd. Clín. Condes. 2016; 27(2): 171-178. https://doi.org/10.1016/j.rmclc.2016.04.006

15. Ebrahimi-Mamaghani M, Saghafi-Asl M, Pirouzpanah S, Aliasg-harzadeh A, Aliashrafi S, Rezayi N, et al. Association of insulinresistance with lipid profile, metabolic syndrome, and hormonal aberrations in overweight or obese women with polycysticovary syndrome. J Health Popul Nutr. 2015; 33:157-67.

16. González A, Gómez JE, Argueta S, Mejía P, Sánchez M. Guía de la práctica clínica de Síndrome metabólico. Asociación Latinoamericana de Diabetes (ALAD). Revista ALAD. 2019; 1-28.

17. Nieto OA. González MM, Rodríguez L. Prevalence and Cardiovascular Risk Factors at a University Community in Armenia-Colombia. International Journal of Collaborative Research on Internal Medicine \& Public Health. 2016; 8(9):572-591.

18. Gmatoid L, Alegre-Diaz J, Wade R, Ramírez-Reyes R, Tapia-Conver R, Gascilazo-Avila A, et al. General and abdominal adiposity and mortality in Mexico City: Prospective Study of 150000 adults. Annals of internnal medicine. 2019; 171(6):397-405. https://doi.org/10.7326/M18-3502 